\title{
Cerebral activation pattern in primary writing tremor
}

\author{
D Berg, C Preibisch, E Hofmann, M Naumann
}

\begin{abstract}
Objective-To compare the cerebral activation pattern during writing of patients with writing tremor with healthy controls using functional MRI

Methods-Three patients with writing tremor and 10 healthy controls were examined using a 1.5 Tesla scanner. All subjects performed a paradigm of alternating 30 second periods of rest or writing. For functional imaging 60 EPI multislice data sets were acquired. All images were analyzed using SPM96 software. Data were analyzed for the group of patients with writing tremor and compared with those of the control group.

Results-Both patients with writing tremor and controls showed a significant activation of the contralateral primary sensorimotor cortex, SMA, and area 44. By contrast, motor cortex activation in writing tremor also included the contralateral premotor area (area 6) and ipsilateral prefrontal area (inferior frontal gyrus; areas 10, 44, and 47). Only patients with writing tremor showed a bilateral activation of the parietal lobule (area 40) with a more pronounced activation on the contralateral side. Furthermore, there was a bilateral activation of the cerebellum with a more pronounced area of activation on the ipsilateral side.

Conclusions-Brain areas activated in writing tremor included activation patterns otherwise typical for both essential tremor and writer's cramp. Therefore a distinct category for writing tremor integrating hallmarks of essential tremor and writer's cramp is proposed.

(F Neurol Neurosurg Psychiatry 2000;69:780-786)
\end{abstract}

Neurology, Bayerische

Julius-Maximilians-

Universität Würzburg,

Josef-Schneider-Strasse

11, D - 97080

Würzburg, Germany

D Berg

M Naumann

Department of

Neuroradiology

C Preibisch

E Hofmann

Correspondence to:

Dr Markus Naumann,

naumann@

mail.uni-wuerzburg.de

Received 21 February 2000 and in revised form

5 July 2000

Accepted 11 July 2000
Keywords: writing tremor; functional magnetic resonance imaging; cerebral activation pattern

Writing tremor is a rare task specific tremor which occurs only during writing ${ }^{12}$ or when the hand adopts a writing position. ${ }^{3}$

Although symptomatic cases of writing tremor after lesions of the central (parietal region $)^{45}$ and peripheral nervous system ${ }^{36}$ have been reported, the pathophysiology of this movement disorder is still unknown. Writing tremor is either regarded as a focal form of essential tremor ${ }^{3-10}$ or as a variant of focal task specific dystonia (writer's cramp). ${ }^{811-13}$

Functional neuroimaging techniques are a valuable tool to study functional anatomy. Although several studies have been performed on the activation pattern in essential tremor and writer's cramp imaging data on writing tremor are sparse.

In essential tremor at rest PET studies demonstrated increased activation in medullary structures, the thalamus, ${ }^{14}$ and bilaterally within the cerebellum leading to a further bilateral increase during unilateral involuntary postural hand tremor. ${ }^{15-19}$ In other studies an activation of the contralateral thalamus, striatum, sensorimotor, mesial, and lateral premotor cortex, as well as the red nucleus and parietal cortex (area 40) was seen bilaterally. ${ }^{17-19} \mathrm{~A}$ single fMRI study during postural tremor in patients with essential tremor reports on a mainly contralateral activation of the primary motor and sensory areas, the globus pallidus, and thalamus, and a bilateral activation of the nucleus dentatus, the cerebellar hemispheres, and the red nucleus. ${ }^{20}$

In patients with writer's cramp, PET studies during writing showed a reduced activation of the contralateral primary motor cortex and enhanced activation of the frontal association cortex..$^{21}{ }^{22}$ Additionally an increased activation of the left thalamus and the ipsilateral more than of the contralateral cerebellum was found..$^{22}$

In writing tremor, hitherto there is only one PET activation study of six patients performing the task of holding a pen to a paper but not writing. ${ }^{24}$ Here an abnormal bilateral cerebellar activation pattern was detected. ${ }^{24}$

In the present study, using fMRI we compared the cerebral activation pattern of patients with writing tremor during writing with a group of control subjects. The cerebrum was scanned from the lower brainstem to the vertex.

\section{Patients and methods}

SUBJECTS AND EXPERIMENTAL DESIGN CONDITIONS

After giving informed consent four patients (one woman, aged 68 years; three men, aged 41,56 , and 68 years) with definite writing tremor participated in the study. The data from the 68 year old man had to be excluded as the fMRI was overshadowed by tremor artifacts. The female patient had had writing tremor for 6 years and reported an additional slight tremor of the right hand during car driving and eating. The 41 year old male patient had recognised the first symptoms of writing tremor 11 years ago and a slight tremor when grasping small objects such as a spoon. Both showed no dystonic postures during writing. In the 56 year old male patient with writing tremor for 9 years, writing tremor was associated with a slight hyperextension of the 
Table 1 Clusters activated during writing in patients with writing tremor

\begin{tabular}{|c|c|c|c|c|c|}
\hline Side & $\begin{array}{l}\text { Number of } \\
\text { voxels in cluster }\end{array}$ & $\begin{array}{l}\text { Maximum } Z \\
\text { score in cluster }\end{array}$ & $\begin{array}{l}\text { Talairach coordinates } \\
(x, y, z) \text { of maximal } \\
\text { peak activation }(\mathrm{mm})\end{array}$ & Region of cluster activation * & $\begin{array}{l}\text { Brodmann area of } \\
\text { peak activation }\end{array}$ \\
\hline Right & 1846 & 8.22 & $24,-58,-24$ & Cerebellum (hemisphere) & \\
\hline Right & & 7.65 & $6,-70,-32$ & Cerebellum (hemisphere) & \\
\hline Right & & 7.15 & $8,-58,-22$ & Cerebellum (superior hemisphere) & \\
\hline Left & 1963 & 7.49 & $-30,-32,50$ & Precentral gyrus & 4 \\
\hline Left & & 7.09 & $-40,-46,60$ & Inferior parietal lobule & 40 \\
\hline Left & & 6.85 & $-24,-22,66$ & Precentral gyrus & 6 \\
\hline Right & 402 & 7.43 & $44,-44,58$ & Inferior parietal lobule & 40 \\
\hline Right & & 5.81 & $54,-34,54$ & Postcentral gyrus & 40 \\
\hline Right & & 5.65 & $40,-54,48$ & Inferior parietal lobule & 40 \\
\hline Right & 956 & 7.31 & $4,-4,64$ & Medial frontal gyrus & 6 \\
\hline Right & & 7.04 & $10,6,42$ & Superior frontal gyrus & 32 \\
\hline Left & & 6.78 & $-4,-22,50$ & Paracentral lobule & 6 \\
\hline Left & 251 & 7.26 & $-58,14,28$ & Inferior frontal gyrus & 44 \\
\hline Left & & 5.17 & $-54,12,-8$ & Superior temporal gyrus & 38 \\
\hline Right & 780 & 7.21 & $54,20,-2$ & Inferior frontal gyrus & 47 \\
\hline Right & & 6.74 & $42,16,-2$ & Inferior frontal gyrus & 47 \\
\hline Right & & 6.69 & $58,14,22$ & Inferior frontal gyrus & 44 \\
\hline Left & 122 & 6.94 & $-34,-56,-36$ & Cerebellum (hemisphere) & \\
\hline Left & & & $-24,-68,-32$ & Cerebellum (hemisphere) & \\
\hline Right & 138 & 6.39 & $44,50,-2$ & Inferior frontal gyrus & 10 \\
\hline
\end{tabular}

Areas activated in patients with writing tremor during writing.

*First order maxima; italics=second order maxima within cluster. The Talairach coordinates correspond to the stereotactic conventions of the atlas of Talairach and Tournoux. ${ }^{30}$

wrist and abduction in the shoulder. All patients were right handed with no neurological abnormalities except for writing tremor and had no dystonic movements in the right arm while at rest. Brain MRI of the female patient displayed small vascular lesions of the right temporal lobe; MRI of all other subjects was normal. There was no previous neuroleptic medication and no family history for movement disorders. None of the patients received any medication at the time of fMRI that could interfere with central activities.

The control group consisted of 10 healthy subjects (five women, five men, mean age: 34.1 years, range: $28-43$ years).

Patients and controls were asked to either rest or write with their right hand according to the instructions given. Each patient wrote the same sentence ("keine Rose ohne Dornen" (no rose without thorns)) to make sure that writing was performed as a complex but automatic and overlearned motor task requiring no decision making. They were trained before the investigation to write in a supine position with eyes closed and as little movement of the arm and head as possible.

The paradigm consisted of alternating a 30 second period of rest and a 30 second period of writing. The rest/writing cycle was repeated three times. Every 3 seconds the subjects were instructed to either write or rest.
MRI DATA ACQUISITION

All experiments were performed on a 1.5 Tesla Magnetom VISION (Siemens, Erlangen, Germany) whole body MRI system equipped with a circular polarised volume head coil. Initially a set of localised images was acquired to position the imaging slices.

For functional imaging 60 EPI multislice data sets were acquired (TE $45 \mathrm{~ms}$, TR 3 seconds, flip angle $90^{\circ}$, acquisition time for the whole paradigm 3 minutes). Each multislice data set contained 16 transverse slices (slice thickness 5 $\mathrm{mm}$, interslice gap $2.5 \mathrm{~mm}$, matrix $64 \times 64$, FOV $25 \mathrm{~cm})$. At the beginning of the session for each subject a set of $16 \mathrm{~T} 1$ weighted anatomical images was acquired in the same slice positions.

\section{DATA ANALYSIS}

All images were analysed using SPM96 Software (Wellcome Department of Cognitive Neurology, London, UK; available at http:// www.fil.ion.ucl.uk)..$^{25-27}$ The first three data sets of each time series were discarded to eliminate the influence of magnetic saturation effects. All remaining EPI volumes were then realigned to the first volume ${ }^{28}$ to correct for movement between scans. This spatial transformation is essentially a six parameter rigid body transformation (three translations, three rotations). To ensure that the structural and functional images were in the same anatomical

Table 2 Clusters activated during writing in control subjects

\begin{tabular}{llllll}
\hline Side & $\begin{array}{l}\text { Number of } \\
\text { voxels in cluster }\end{array}$ & $\begin{array}{l}\text { Maximum } Z \\
\text { score in cluster }\end{array}$ & $\begin{array}{l}\text { Talairach coordinates } \\
(x, y, z) \text { of maximal } \\
\text { peak activation }(\mathrm{mm})\end{array}$ & Region of cluster activation & $\begin{array}{l}\text { Brodmann area } \\
\text { of peak } \\
\text { activation }\end{array}$ \\
\hline Right & 1625 & 8.07 & $24,-52,-24$ & Cerebellum (hemisphere, dentate nucleus) & 2 \\
Left & 2222 & 7.68 & $-42,-28,36$ & Postcentral gyrus & 7 \\
Left & & 7.45 & $-26,-60,58$ & Superior parietal lobule & 4 \\
Left & & 7.44 & $-42,-14,48$ & Precentral gyrus & 44 \\
Left & 94 & 6.55 & $-54,12,18$ & Inferior frontal gyrus & 32 \\
& 193 & 6.35 & $0,4,46$ & Cingulate gyrus & 6 \\
\end{tabular}

Areas activated in patients with writer's cramp during writing.

$\star$ First order maxima; italics=second order maxima within cluster. The Talairach coordinates correspond to the stereotactic conventions of the atlas of Talairach and Tournoux. ${ }^{30}$ 
space, the $\mathrm{T} 1$ weighted anatomical images were coregistered to a mean EPI data set which was previously calculated from the realigned time series. ${ }^{28}$ Finally, the anatomical image was spatially normalised ${ }^{28}$ to a standard template, ${ }^{29} 30$ using a 12 parameter affine transformation. The same transformation was then applied to the EPI time series data. The
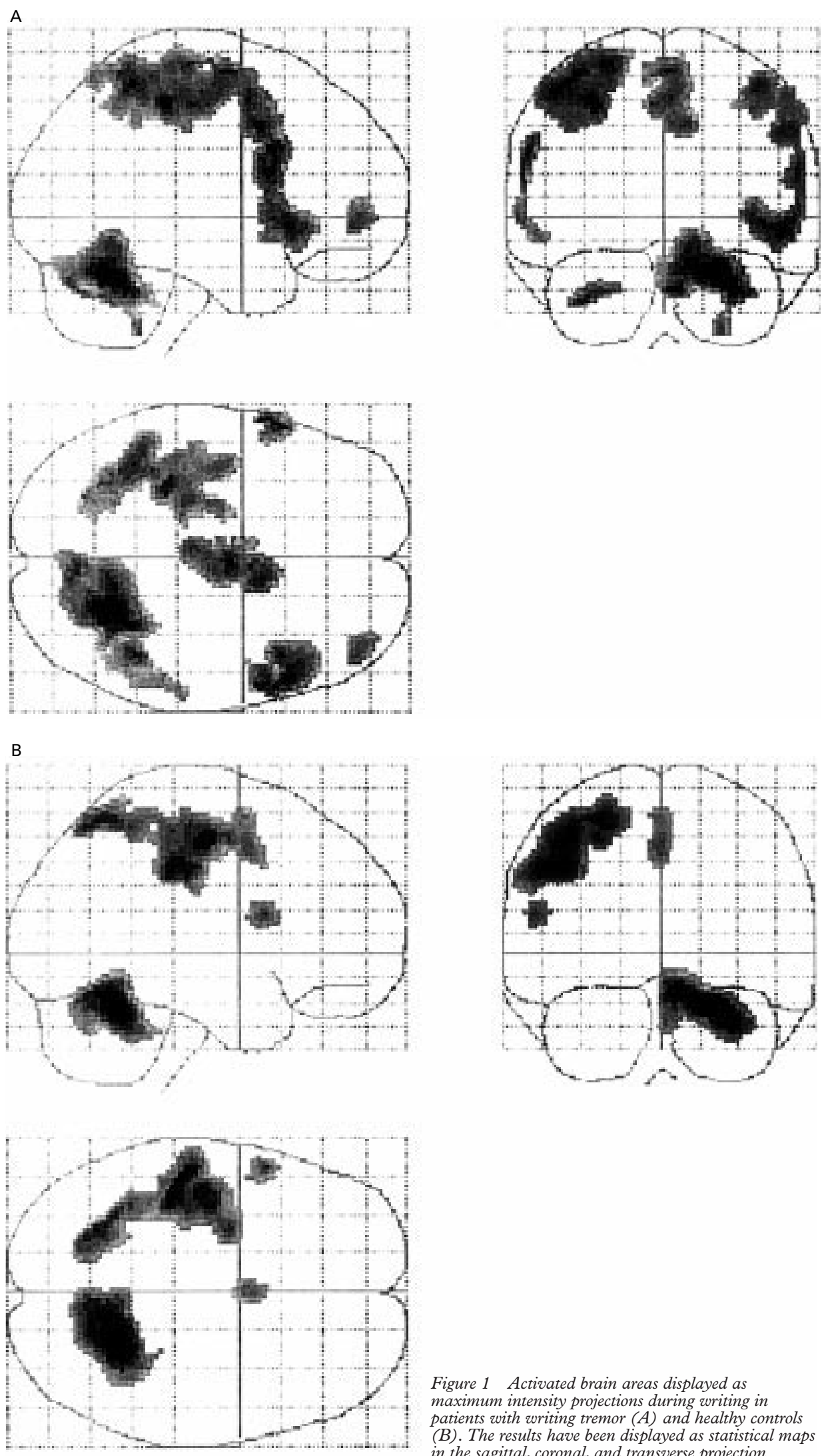

Figure 1 Activated brain areas displayed as maximum intensity projections during writing in patients with writing tremor $(A)$ and healthy controls (B). The results have been displayed as statistical maps in the sagittal, coronal, and transverse projection according to the atlas of Talairach and Tournoux. ${ }^{30}$ 
images were smoothed with a Gaussian kernel of $10 \mathrm{~mm}$ full width at half maximum (FWHM) for single subject and group analy-
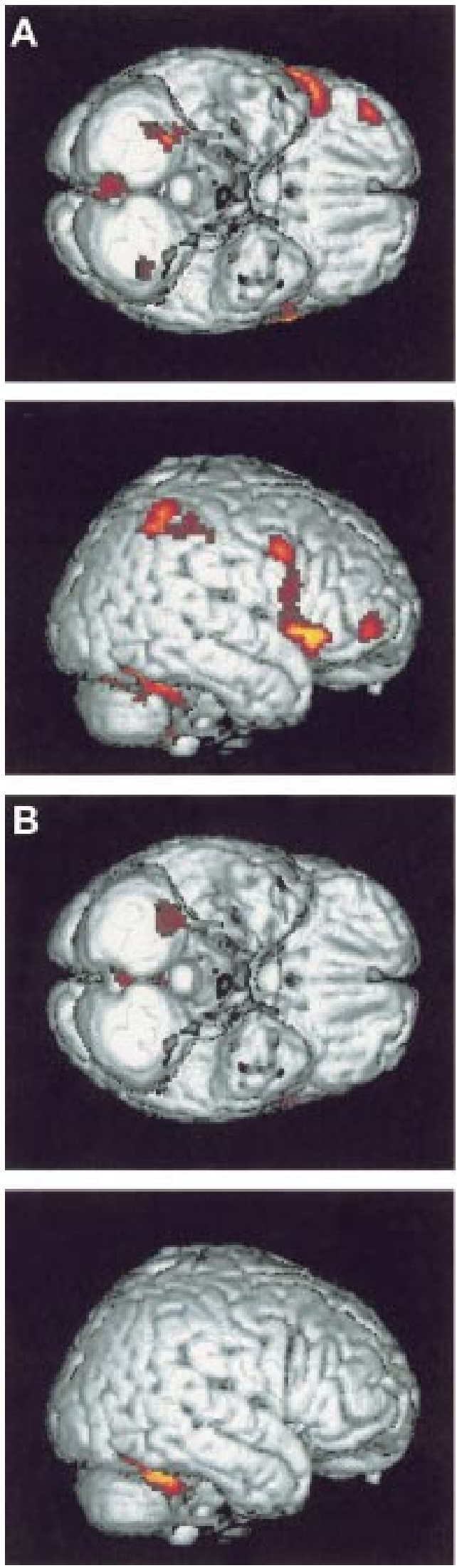

Figure 2 Activation maps projected on the surface of the brains in patients with writing tremor $(A)$ and healthy controls (B). sis. The images were proportionally scaled to remove the effect of global signal differences between subjects.
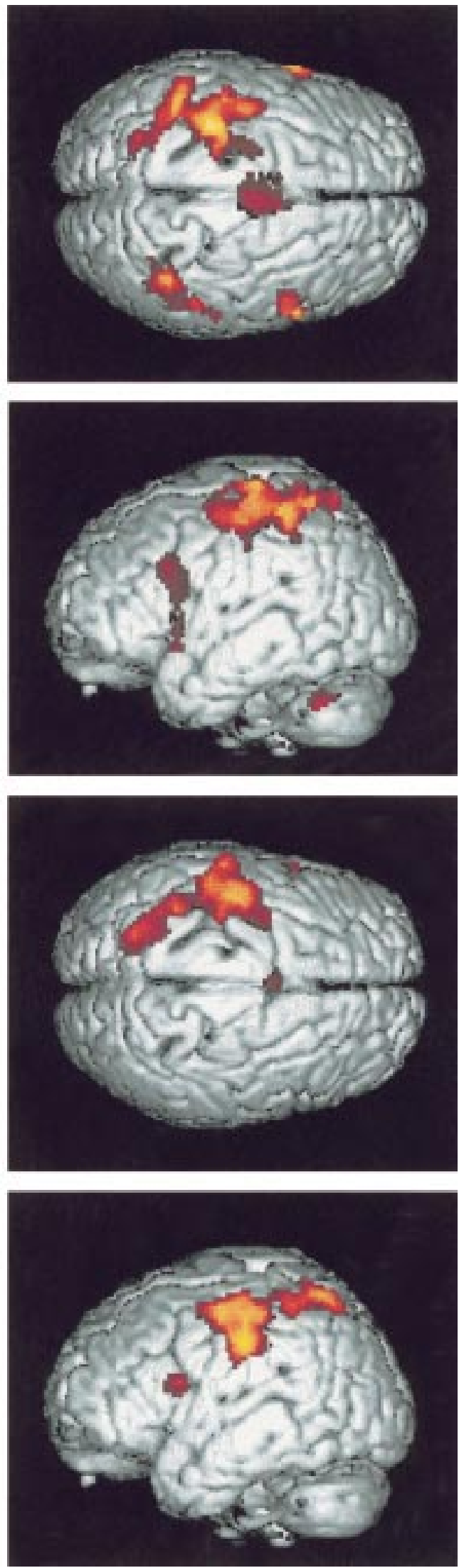

from the bottom and the top as well as of both hemispheres 
STATISTICAL ANALYSIS

Statistical analysis was performed using the general linear model as employed by SPM96. The different conditions (rest and writing) were modelled as boxcar functions convolved with the haemodynamic response function. ${ }^{26}$ Aliased low frequency noise caused by cardiac and respiratory effects were modelled using cosine waves of 120 to 240 seconds. The remaining autocorrelation of the residual errors was removed by temporal smoothing with a 2.8 second FWHM gaussian kernel. Statistical maps ( $Z$ statistics) were created by applying appropriate contrasts to the parameter estimates for each condition. The contrast used was +1 for writing and -1 for rest. This contrast shows voxels that have a significantly larger signal during writing relative to rest. The resulting statistical parametric maps were interpreted by referring to the gaussian random field theory because of the need to correct for multiple independent comparisons. Data were analyzed for the group of subjects with writing tremor and for the group of healthy volunteers separately. As only data of three patients with writing tremor were analyzed, no group comparison was performed for patients with writing tremor.

\section{Results}

Except for small vascular lesions of the right temporal lobe in one patient, MRI of all subjects investigated was normal. The field of view comprised the whole cerebrum and the cerebellum.

Data of activated areas (statistical and anatomical data) of patients with writing tremor are given in table 1 and for the control group in table 2 . The tables summarise the coordinates of first order maxima of activated clusters as well as the second order maxima within clusters, which are especially important in clusters covering large areas. The statistics (height threshold $\mathrm{p}=0.00001$, extent threshold $\mathrm{p}=0.001$ ) are displayed as maximum intensity projections on a "glass brain" in normalised Talairach space: figure $1 \mathrm{~A}$ and $\mathrm{B}$ show the activated brain areas of patients with writing tremor and controls. Figure $2 \mathrm{~A}$ and B display the respective activation maps projected on the surface of the brain.

PATIENTS WITH WRITING TREMOR AND CONTROLS Patients with writing tremor and controls in common showed a marked activation of the left sensorimotor cortex (SMC) with a remarkably consistent pattern, of area 44, and of the right cerebellar hemisphere, which was larger in patients with writing tremor.

By contrast, patients with writing tremor in addition had a marked activation of the left cerebellar hemisphere. In patients with writing tremor, the activated SMC area extended further caudal and anterior from the primary motor cortex towards the premotor cortex (area 6). This relative overactivity of the motor cortex in writing tremor was also reflected by the peak activation of the sensorimotor cluster in writing tremor, which is located anterior to that of healthy volunteers. Only patients with writing tremor showed an activation of the ipsilateral prefrontal cortex (inferior frontal gyrus; areas 44, 47, and 10) and bilateral parietal lobule (area 40) with a more pronounced activation on the contralateral side.

\section{Discussion}

This fMRI study shows that activation patterns of the cerebellum, parietal lobe, and frontal cortex are different in patients with primary writing tremor compared with controls during the task of writing.

CEREBELLAR, PARIETAL, AND FRONTAL LOBE ACTIVATION IN PRIMARY WRITING TREMOR The bilateral cerebellar activation in writing tremor underscores the pivotal role of the cerebellum in tremor generation already known from patients with essential tremor. ${ }^{15-19}$ A bilateral cerebellar activation in unilateral postural essential tremor has been demonstrated in PET studies. ${ }^{17}{ }^{31}$ Interestingly, bilaterally increased cerebellar rCBF was already visible in patients with essential tremor at rest, ${ }^{16-19}$ which led to a further bilateral increase in the presence of unilateral involuntary postural tremor, but not during passive wrist oscillation or during voluntary arm extension by control subjects. $^{15-19}$ Moreover, ethanol in alcohol responsive patients with essential tremor ${ }^{19}$ and stimulation of the contralateral ventral intermediate nucleus (Vim) of the thalamus in patients with unilateral tremor dominant Parkinson's disease ${ }^{32}$ have led to a significant decrease of previously bilaterally increased cerebellar blood flow. In support of the results of our study a bilateral cerebellar activation has also been described in a previous PET study in patients with writing tremor holding a pen to a paper. ${ }^{24}$ Therefore a bilateral hyperactivity of the cerebellar networks seems to play an important part also in the pathophysiology of writing tremor. Although patients with writer's cramp and healthy subjects may show a bilateral cerebellar activation during the task of writing, this activation is usually less pronounced compared with patients with essential tremor or writing tremor. ${ }^{22}$

The cerebellum has been shown to be involved in preparatory and executive activity $^{3334}$ and different aspects of cognitive behaviour and affective states. ${ }^{35}$ It receives input from multiple areas of the cortex including motor, premotor, and parietal areas, conveyed by way of several parallel pathways. ${ }^{35}$ The output targets project to various cortical areas such as the premotor, prefrontal, and posterior parietal cortex. ${ }^{35-40}$ These widespread connections suggest that a bilateral cerebellar activity should also be reflected by increased neuronal activity at a cortical level which indeed could be seen as an increased bilateral activation of the inferior parietal lobules. However, biparietal overactivity might not only be the result of an activated bilateral cerebellar loop. It may also reflect increased intrinsic activity of the parietal lobes resulting from an increased task difficulty and effort to perform the task as it is particularly the case in writing tremor. The parietal cortex is reciprocally connected with various isocorti- 
cal areas of the frontal, temporal, and occipital lobe ${ }^{41}$ and is involved in the sequential processing of somatotopically organised information received from the adjacent primary somatosensory cortices. ${ }^{35}$ The inferior parietal lobule corresponds to the secondary somatosensory area and has bilateral but contralateral dominant receptive fields. ${ }^{42} \mathrm{~A}$ bilateral activation of secondary somatosensory areas during active movements has recently been associated with the sensorimotor integration using proprioreceptive feedback and auditory cues ${ }^{43-45}$ and with increased attention. ${ }^{43}$ The increased activation of the ipsilateral SMA could be an additional result of this bilaterally active loop, as it receives major input from the posteroparietal cortex. ${ }^{41}$

The prefrontal cortex is important for kinaesthetic, motivational, and spatially related functions. In particular, the right prefrontal cortex is activated in subjects choosing either direction or timing of movements. ${ }^{47-49}$ The activation of the right prefrontal area only seen in patients with writing tremor may therefore reflect the higher neuronal activity required for directing timing of movements under severely disabled circumstances.

Artificial activation patterns as the consequence of tremor in our patients have to be discussed but are very unlikely as motion artifacts were detected by the software, which led to the exclusion of one patient. Furthermore, artifacts would be expected to be scattered all over the brain with a predominance at the vertex. The activated areas shown in the present study clearly correspond to brain areas which are physiologically activated during writing and which can be easily integrated in the pathophysiological concept of tremor disorders.

\section{ACTIVATION PATTERN IN WRITING TREMOR}

COMPARED WITH WRITER'S CRAMP AND

ESSENTIAL TREMOR

There are several similarities in the activation pattern of patients with writing tremor with patients with writer's cramp and essential tremor. The findings of an increased activation of premotor cortical areas and the bilateral but predominantly contralateral cerebellar activation in our patients with writing tremor conform to previous PET studies on patients with writer's cramp. ${ }^{21}{ }^{22}$ This points towards a common aetiology of writer's cramp and writing tremor, which is also supported by clinical and electrophysiological findings: both writer's cramp and writing tremor are focal and task specific with a coexistence of focal tremor and dystonia in some affected persons ${ }^{50}{ }^{51}$ and even within the same family. ${ }^{53}$ In addition, cocontractions of antagonist muscles in the forearm which produce dystonic movements have been seen clinically and were also evident in EMG recordings in both conditions. ${ }^{11} 13$

A bilateral parietal and increased bilateral cerebellar activation has been reported in previous PET studies in patients with essential tremor. ${ }^{17} 1824$ Particularly, the marked biparietal activation is in line with the hypothesis of writing tremor being a focal variant of essential tremor. This hypothesis is further underscored by various clinical findings such as similar frequencies in both conditions, and the response to propanolol, alcohol, and Vim stimulation. 1371054

In conclusion, writing tremor shares several clinical aspects and the pattern of the cortical activation with both writer's cramp and essential tremor, as measured by fMRI and PET. Therefore, writing tremor cannot easily be classified as a variant of either essential tremor or writer's cramp. A distinct category should be proposed integrating hallmarks of both entities.

1 Rothwell JC, Traub MM, Marsden CD. Primary writing tremor. F Neurol Neurosurg Psychiatry 1979;42:1106-14.

2 Deuschl G, Bain P, Brin M, et al. Consensus statement of the movement disorder society on tremor. Mov Disord 1998;13:2-23

3 Bain PG, Findley LJ, Britton TC, et al. Primary writing tremor. Brain 1995;116:203-9.

4 Milanov I, Georgiev D. Writer's cramp and tremor due to brain tumor. Can $\mathcal{F}$ Neurol Sci 1995;22:59-61.

5 Kim JS, Lee MC. Writing tremor after discrete cortical infarction. Stroke 1994;25:2280-2.

6 Soland VL, Bhatia KP, Volonte MA, et al. Focal task-specific tremors. Mov Disord 1996;11:665-70.

7 Kachi T, Rothwell JC, Cowan JMA, et al. Writing tremor: its relationship to benign essential tremor. $\mathcal{F}$ Neurol Neurosurg Psychiatry 1985;48:545-50.

8 Rosenbaum F, Jankovic J. Focal task-specific tremor and dystonia. Neurology 1988;38:522-7.

9 Jimenez-Jimenez FJ, Cabrera-Valdivia F, Orti-Pareja M, et al. Bilateral primary writing tremor. Eur $f$ Neurol 1998;5:613-4.

10 Koller WC, Martyn B. Writing tremor:its relationship to essential tremor. F Neurol Neurosurg Psychiatry 1986;49: 220.

11 Ravits J, Hallett M, Baker M, et al. Primary writing tremor and myoclonic writer's cramp. Neurology 1985;35:1387-91. 12 Cohen LG, Hallett M, Sudarsky L. A single family with writer's cramp, essential tremor and primary writing tremor. Mov Disord 1987;2:109-16.

13 Elble RJ, Moody C, Higgins C. Primary writing tremor. Mov Disord 1990;5:118-26.

14 Hallett M, Dubinsky RM. Glucose metabolsim in the brain of patients with essential tremor. $\mathcal{F}$ Neurol Sci 1993;114:4558 .

15 Brooks DJ, Jenkins IH, Bain P, et al. A comparsion of abnormal patterns of cerebral activation associated with neuropathic and essential tremor. Neurology 1992;42(suppl 3):423.

16 Zeffiro TA, Liao KK, Hallett M. Regional cerebral blood flow abnormalities in essential tremor. Neurology 1992; 42(suppl 3):423.

17 Jenkins IH, Bain PG, Colebatch JG, et al. A positron emission tomography study of essential tremor: evidence for sion tomography study of essential tremor: evidence for
overactivity of cerebellar connections. Ann Neurol 1993;34: overactivit $82-90$.

18 Wills AJ, Jenkins IH, Thompson PD, et al. Red nuclear and cerebellar but no olivary activation associated with essential tremor: a positron emission tomography study. Ann Neurol 1994;36:636-42.

19 Boecker H, Wills AJ, Ceballos-Baumann A, et al. The effect of ethanol on alcohol-responsive essential tremor: a positron emission tomography study. Ann Neurol 1996;39: $650-8$.

20 Bucher SF, Seelos KC, Dodel RC, et al. Activation mapping in essential tremor with functional magnetic resonance imaging. Ann Neurol 1997;41:32-40.

21 Ceballos-Baumann AO, Sheean G, Passingham RE, et al. Botulinum toxin does not reverse the cortical dysfunction associated with writer's cramp. A PET study. Brain 1997;120:571-82.

22 Odergren T, Stone-Elander S, Ingvar M. Cerebral and cerebellar activation in correlation to the action-induced dystonia in writer's cramp. Mov Disord 1998;13:497-508.

23 Rao SM, Binder JR, Bandettini PA, et al. Functional Rao SM, Binder JR, Bandettini PA, et al. Functional magnetic resonance imaging of co
ments. Neurology 1993;43:2311-18.

24 Wills AJ, Jenkins IH, Thompson PD, et al. A positron emission tomography study of cerebral activation associated with essential and writing tremor. Arch Neurol 1995;52: 299-305.

25 Friston KJ, Holmes AP, Poline J-B, et al. Analysis of fMRI time-series revisited. Neuroimage 1995;2:45-53.

26 Friston KJ, Holmes AP, Worsley KP, et al. Statistical parametric maps in functional imaging: a general linear approach. Hum Brain Mapp 1995;2:189-210.

27 Worsley KP, Friston KJ. Analysis of fMRI time-series revisited - again [comment]. NeuroImage 1995;2:173-81.

28 Friston KJ, Ashburner J, Frith CD, et al. Spatial registration and normalization of images. Hum Brain Mapp 1995;2:125 .

29 Evans AC, Collins DL. A 305-member MRI-based stereotaxic atlas for CBF activation studies. $\mathcal{F} \mathrm{Nucl} \mathrm{Med}$ 1993;34:70-1.

30 Talairach P, Tournoux J. A stereotaxic co-planar atlas of the human brain. Stuttgart: Thieme, 1988. 
31 Colbatch JG, Findley LJ, Frackowiak RS, et al. Preliminary report: activation of the cerebellum in essential tremor. report: activation of the

32 Deiber MP, Pollak P, Passingham R, et al. Thalamic stimulation and supression of parkinsonian tremor: evidence of a cerebellar deactivation using positron emission tomography. Brain 1993;116:267-79.

33 Houk JC, Buckingham JT, Barto AG. Models of the cerebellum and motor learning. Behav Brain Sci 1996;19: 368-83.

34 Verleger R, Wascher E, Wauschkuhn B, et al. Consequences of altered cerebellar input for the cortical regulation of motor coordination, as reflected in EEG potentials. Exp Brain Res 1999;127:409-22.

35 Schmahmann JD, Pandya DN. The cerebrocerebellar system. Int Rev Neurobiol 1997;41:31-60.

36 Sasaki K, Oka H, Matsuda Y, et al. Electrophysiological studies of the projections from the parietal association area studies of the projections from the parietal association area

37 Sasaki K. Electrophysiological studies on the cerebellothalamocortical projections. Applied Neurophysiology 1976-77; lamocortica

38 Middleton FA, Strick PL. Cerebellar output channels. Int Riddleton FA, Strick PL. Cerebrobiol 1997;41:61-82.

39 Shinoda Y, Izawa Y, Sugiuchi Y, et al. Functional significance of excitatory projections from the precerebella nuclei to interpositus and dentate neurons for mediating motor, premotor and parietal cortical inputs. Prog Brain Res 1997;114:193-207.

40 Matelli M, Luppino G, Rizzolatti G. Convergence of pallidal and cerebellar outputs on the frontal motor areas. Acta Biomed Ateneo Parmense 1995;66:83-92.

41 Paxinos $\mathrm{G}$, ed. The human nervous system. San Diego, California: Academic Press, 1990.

42 Burton H. Second somatosensory cortex and related areas. In: Jones EG, Peters A, eds. Cerebral cortex. Vol 5. New York: Plenum Press, 1986:31-98.
43 Mima T, Sadato N, Yazawa S, et al. Brain structures related to active and passive finger movements in man. Brain 1999;122:1989-97.

44 Mattingley JB, Huasain M, Rorden C, et al. Motor role of human inferior parietal lobe revealed in unilateral neglect patients. Nature 1998;392:179-82.

45 Snyder LH, Batista AP, Andersen EA. Coding of intention in the posterior parietal cortex. Nature 1997;386:167-70.

46 Mima T, Nagamine T, Nakamura K, et al. Attention modulates both primary and second somatosensory cortical activities in humans: a magnetoencephalographic study. $\mathscr{f}$ Neurophysiol 1998;80:2215-21.

47 Samuel M, Williams SCR, Leigh PN, et al. Exploring the temporal nature of hemodynamic response of cortical motor areas using functional MRI. Neurology 1998;51: 15675

48 Playford ED, Jenkins IH, Passingham RE, et al. Impaired mesial frontal and putamen activation in Parkinson's disease: a positron emission tomography study. Ann Neurol 1992 32:151-61.

49 Jahanshahi M, Jenkins IH, Brown RG, et al. Self-initiated versus externally triggered movements $\mathrm{I}$. an investigation using measurement of regional cerebral blood flow with PET and movement related potentials in normal and Parkinson's disease subjects. Brain 1995;118:913-33.

50 Sheehy MP, Marsden CD. Writer's cramp: a focal dystonia. Brain 1982;105:461-80.

51 Dubinsky RM, Gray CS, Koller WC. Essential tremor and dystonia. Neurology 1993;43:2382-84.

2 Lang AE. Writing tremor and writing dystonia. Mov Disord 1990;5:354.

53 Hayashi M, Koide H. Idiopathic torsion dystonia and writing tremor within a family. Brain Dev 1997;19:556-8.

54 Ohye C, Miyazaki M, Hirai T, et al. Primary writing tremor treated by stereotactic selective thalamotomy. $\mathcal{F}$ Neurol Neurosurg Psychiatry 1982;45:988-97.

\section{André Chantemesse (1851-1919)}

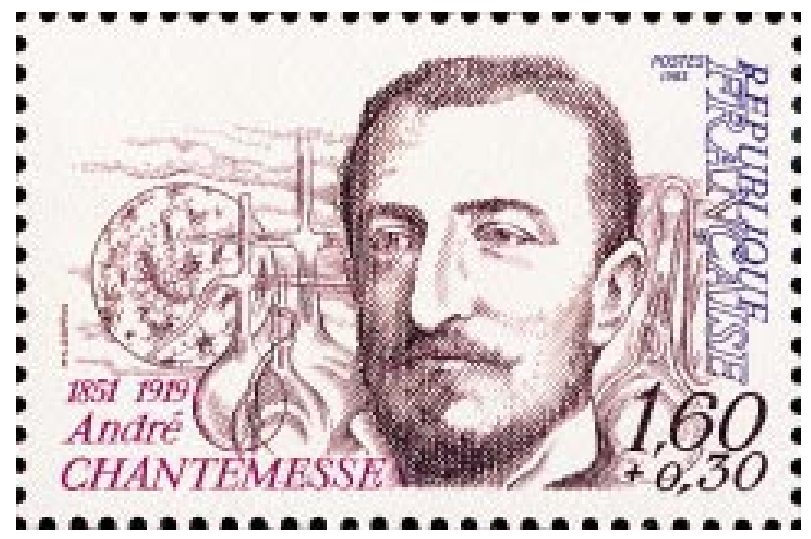

Chantemesse was a French bacteriologist who became a colleague of Pasteur with his thesis on tuberculous meningitis. He began studying bacteriology with Georges Widal. Using animal experiments during work with Widal he succeeded in preventing typhoid. Chantemesse's other work included culture of the dysentery bacillus, vaccination of humans, and bacteriological examination of water. He was a physician in Paris, later Professor at the medical academy, and chief hygienic technician of the Ministry of Home Affairs. Chantemesse was honoured philatelically by France in 1982 (Stanley Gibbons 2515, Scott B543).

L F HAAS 